\title{
Circuito cerrado para una industria sostenible, aportaciones del sector cementero
}

\author{
Closed circuit for a sustainable industry, cement sector \\ contribution
}

\author{
A. Zaragoza ${ }^{(*)}$
}

\section{RESUMEN}

La búsqueda del desarrollo sostenible para la industria sólo puede entenderse desde un enfoque global que abarque tanto la actividad industrial como el producto. La actividad industrial necesita incorporar al proceso productivo soluciones que minimicen su impacto reduciendo el consumo de recursos y las emisiones a la atmósfera. El producto debe incorporar características sostenibles tales como la posibilidad de ser reciclado y/o valorizado en algún momento de su ciclo de vida y, así, poder ser reintroducido de nuevo en la actividad industrial.

De esta manera, el producto se convierte en la piedra angular de un sistema de producción en "circuito cerrado" que implique a todos los sectores industriales en el concepto de reciclaje de productos y residuos y que minimice la huella del proceso. El sector cementero debe seguir incidiendo en las posibilidades de reciclado de los materiales fabricados con cemento, además de los utilizados propiamente en su fabricación, y, sobre todo, se debe seguir profundizando en la relación entre Ciclo de Vida y reciclaje, lo que proporcionará al sector una metodología y unas herramientas útiles para reducir el impacto ambiental y reforzar sus contribuciones sociales y económicas.

Por ello el sector está haciendo esfuerzos para la puesta en marcha de una industria en circuito cerrado en la que se reutilicen materiales y energía, minimizando al máximo su afección en el entorno, a la vez que se está trabajando en una conceptualización de este modelo de producción en cadena, que implique a todos los sectores industriales en el reciclado de materiales. Prueba de este compromiso son los acuerdos firmados con FER y Sigrauto para el aprovechamiento de vehículos fuera de uso y con UNESID para la valorización de residuos siderúrgicos, así como el proyecto Pressure para la gestión integral de residuos industriales.

Palabras clave: sostenibilidad, residuo, valorización, reciclaje, cemento.

\section{SUMMARY}

The search of a sustainable development for the industry can only be understood from a global approach which takes account of both the industrial activity and the product. Industrial activity needs adding new systems to the production process that minimize its impact reducing the consumption of resources and atmospheric emissions. The product must incorporate sustainable features such as the capacity to be recycled and/or valorised throuhgout its life cycle and, so, being reintroduced again in the industrial activity.

This way, product turns into the cornerstone of a "closed circuit" production system that involves all industrial sectors in recycling products and waste and minimizes the frootprint of the process. Cement sector must continue insisting on the development of recycling of cementicious maerials and also on the recycling of materials during its production, and, especially, we must continue studying in depth the relationship between Life Cycle and recycling, which will provide the sector with a methodology and with useful tools to reduce the environmental impact.

Therefore, the sector is making efforts for achieving an industry in "closed circuit", in which materials and energy are re-used, minimizing to the maximum its affection to the environment. At the same time, we are working on the conceptualizing of this production model that involves all the industrial sectors in material recycling. As a reuslt of this commitment, cement industry has signed aggrement of colaboration with FER and Sigrauto to use vehicles out of use and with UNESID to valorise steel waste and also the project Pressure for a global management of industrial waste.

\section{$113-88$}

Keywords: sustainability, waste, valorisation, recycling, cement.

\footnotetext{
${ }^{(* D}$ Director General de Oficemen. Profesor Titular de la Universidad Politécnica de Madrid (España) Persona de contacto/Corresponding author: azaragoza@oficemen.com (A. Zaragoza)
}

Fecha de recepción: 05-1-08 Fecha de aceptación: 03-12-09 


\section{LA SOSTENIBILIDAD Y LA GESTIÓN DE RESIDUOS}

En general, se define la sostenibilidad como la satisfacción de las necesidades del presente sin comprometer las de las generaciones futuras. En este sentido, la gestión de residuos emerge como un punto clave para no agotar los recursos básicos y no dañar el medio natural imprescindible para mantener el desarrollo social y el estado de bienestar. En ambos casos, la solución pasa por un tratamiento adecuado de los residuos de manera que se minimice su impacto sobre el medioambiente a la vez que se reduce el consumo de materias primas.

Es evidente que la industria debe dirigir sus esfuerzos a minimizar la generación de residuos pero, ante la imposibilidad técnica de no producirlos, es imprescindible una correcta gestión de los mismos mediante su reutilización, reciclado y valorización, ya sea material o energética, situándose al final de la cadena de gestión su incineración (con o sin recuperación de energía) o su vertido.

Mediante una correcta gestión de los residuos industriales se completa el ciclo de vida (1) del producto, reincorporándolo al proceso productivo, reduciendo de esta manera el consumo, bien de materias primas naturales, bien de combustibles fósiles.

Es, pues, preciso incidir en dos aspectos: las posibilidades de reciclado de los productos fabricados y la posibilidad de utilizar materiales reciclados en su fabricación. Además, se debe seguir profundizando en la influencia del reciclaje en el Ciclo de Vida, lo que proporcionará a la industria una metodología y unas herramientas útiles para reducir el impacto ambiental, no sólo de su actividad, sino también de las aplicaciones de sus productos. Por eso señalamos como una posible solución la puesta en marcha de una industria en circuito cerrado que minimice al máximo su afección en el entorno.

\section{UNA NUEVA FILOSOFÍA: INDUSTRIA EN CIRCUITO CERRADO}

Actualmente se está fomentando la innovación como la vía para aumentar la sostenibilidad de la producción industrial y se están desarrollando numerosos proyectos sectoriales en esta dirección. Sin embargo, la innovación, y aún menos la innovación sectorial, no es suficiente para garantizar una sostenibilidad real en los procesos industriales. Es una iniciativa que en algunos aspectos se ha quedado pequeña y es necesario dar un paso más y avanzar hacia una producción en circuito cerrado, es decir, una industria en la que en todos los procesos y sectores se puedan vincular, reutilizando los materiales y buscando nuevas autonomías energéticas, evitando así residuos, ahorrando energía y reduciendo las emisiones de Gases de Efecto Invernadero (GEI).

Por lo tanto, si conseguir la integración de todos los procesos productivos es un objetivo, éste no se podrá alcanzar sin realizar un trabajo en común de todos los sectores industriales buscando lograr una menor afección al entorno. De esta forma, el primer paso hacia la sostenibilidad real está en una cooperación en red, sobre todo, en lo referente a la gestión de residuos. Es en esta filosofía de trabajo donde más se aprecia la relación entre el Análisis del Ciclo de Vida y el reciclaje.

La gestión de residuos juega un papel fundamental y, en este campo, las fábricas de cemento presentan grandes posibilidades para aprovechar parte de los residuos minerales generados por otros procesos industriales, no sólo porque éstos tienen una composición similar a la de sus materias primas, sino también por mejorar las prestaciones de los cementos, ya que se pueden añadir como adiciones en la molienda del clínker junto con otros minerales (3). De esta forma se valora el Ciclo de Vida de los productos, empleándolos hasta el final real de sus posibilidades y no desechándolos sin más cuando aún pueden ser de utilidad.

Este proceso se conoce con el nombre de valorización material, una práctica para sustituir materias primas naturales por materiales que están total o parcialmente descarbonatados y así poder reducir las emisiones del proceso natural de fabricación del cemento. La valorización material es una práctica de gran utilidad para el sector dada la gran cantidad de materias primas que consume, tal y como se puede ver en las Figuras 1 y 2.

En este sentido, la única posibilidad para aumentar la sostenibilidad del sector es reducir las 54 millones de toneladas de materias primas naturales utilizadas en 2007 mediante el uso de residuos industriales procedentes de otros sectores, que se utilizarán como materias primas alternativas.

Como se puede observar en la Figura 2, el esfuerzo de la industria del cemento en este sentido está siendo muy importante $y$, desde 1997 se ha incrementado el porcentaje de materias primas alternativas utilizadas en la fabricación de cemento de un 4,5\% a un $9,5 \%$, lo que sitúa la tasa de sustitución en consonancia con las de algunas empresas líderes mundiales (en torno a un 10\%). 


\begin{tabular}{|c|c|c|c|c|c|c|c|c|c|c|c|c|}
\hline \multicolumn{13}{|c|}{ CONSUMO DE MATERIAS PRIMAS (cifras en toneladas) } \\
\hline & & 1998 & 1999 & 2000 & 2001 & 2002 & 2003 & 2004 & 2005 & 2006 & 2007 & 2008 \\
\hline \multirow{20}{*}{ 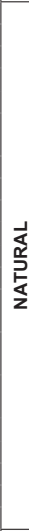 } & ARCILLAS & 2.049 .694 & 2.235 .623 & 2.106 .829 & 2.663 .569 & 3.098 .731 & 3.338 .703 & 3.132 .219 & 2.963 .019 & 2.943 .592 & 2.757 .111 & 2.761 .668 \\
\hline & \begin{tabular}{|l} 
ARENA \\
\end{tabular} & 38.865 & 54.043 & 41.834 & 63.253 & 66.004 & 670.372 & 534.617 & 592.887 & 538.126 & 597.345 & 535.439 \\
\hline & ARENISCA & 948.789 & 905.695 & 803.881 & 636.793 & 783.795 & 237.455 & 187.242 & 202.128 & 283.271 & 234.958 & 162.110 \\
\hline & BAUXITA & 34.039 & 64.263 & 14.059 & 29.655 & 24.284 & 25.197 & 69.331 & 67.855 & 77.084 & 78.228 & 91.734 \\
\hline & CALIZA & 28.570 .925 & 30.232 .938 & 30.588 .901 & 31.482 .863 & 32.024 .546 & 32.888 .394 & 35.232 .174 & 34.881 .014 & 34.802 .077 & 36.615 .254 & 31.195 .132 \\
\hline & $\begin{array}{l}\text { CAOLIN Y ARCILLAS } \\
\text { CAOLINICAS } \\
\end{array}$ & 236.693 & 247.676 & 153.504 & 145.660 & 149.756 & 370.500 & 736.316 & 290.620 & 230.808 & 184.581 & 171.262 \\
\hline & CRETA & 24.206 & 20.930 & 24.035 & 27.175 & 29.376 & 21.781 & & & & & \\
\hline & \begin{tabular}{|l|} 
CUARZO \\
\end{tabular} & & & & & & 3.869 & 5.986 & 5.652 & 3.841 & 3.034 & 3.807 \\
\hline & HIERRO & 16.107 & 9.743 & 12.181 & 23.034 & 39.753 & 77.831 & 61.437 & 59.956 & 73.872 & 79.624 & 305.551 \\
\hline & KIESELBURH & & & 7.700 & 202.260 & 246.567 & 213.543 & 240.450 & 297.384 & 285.363 & 167.084 & 117.214 \\
\hline & LIMONITA & 18.903 & 19.262 & 67.966 & 70.351 & 68.994 & 264.637 & 161.236 & 218.691 & 209.169 & 202.878 & 183.690 \\
\hline & MARGAS & 9.450 .161 & 8.190 .517 & 9.362 .507 & 9.497 .342 & 9.310 .392 & 9.796 .405 & 10.271 .536 & 10.014 .650 & 10.179 .971 & 8.946 .707 & 8.032 .716 \\
\hline & $\begin{array}{l}\text { MINERAL DE YESO Y } \\
\text { ANHIDRITA }\end{array}$ & 1.380 .584 & 1.536 .989 & 1.697 .621 & 1.840 .056 & 1.964 .676 & 1.623 .959 & 1.728 .253 & 1.912.167 & 2.037.476 & 1.942 .919 & 1.519 .632 \\
\hline & $\begin{array}{l}\text { OTRAS MATERIAS PRIMAS } \\
\text { NATURALES }\end{array}$ & 8.680 & 17.959 & 29.610 & 27.706 & 724.654 & 40.120 & 107.286 & 108.335 & 117.734 & 179.225 & 207.755 \\
\hline & $\begin{array}{l}\text { OTRAS SUSTANCIAS } \\
\text { ARCILLOSAS CRUDO } \\
\end{array}$ & & & & & & 2.643 & & & 8.377 & 7.479 & 11.267 \\
\hline & PIRITAS & 340.483 & 356.254 & 319.069 & 326.071 & 288.901 & 234.660 & 206.338 & 206.405 & 169.522 & 129.134 & 82.277 \\
\hline & \begin{tabular}{|l|} 
PIZARRAS \\
\end{tabular} & 398.194 & 434.091 & 390.670 & 365.869 & 407.485 & 502.143 & 391.872 & 334.015 & 489.358 & 408.180 & 418.534 \\
\hline & PUZOLANAS CEMENTO & 960.864 & 896.876 & 946.696 & 980.118 & 863.997 & 773.319 & 804.666 & 898.948 & 1.037 .628 & 882.466 & 579.571 \\
\hline & SERICITA & & 23.173 & 6.598 & 935 & 14.690 & & 2.239 & & & & \\
\hline & SILICE & 32.634 & 39.731 & 116.766 & 7.843 & 6.223 & 1.071 & 3.072 & 6.050 & & 43.308 & 84.644 \\
\hline & Tot & 44.509 .821 & 45.285 .763 & 46.690 .427 & 48.390 .553 & 50.112 .824 & 51.086 .602 & 53.876 .270 & 53.059 .776 & 53.487 .269 & 53.459 .515 & 46.464 .003 \\
\hline \multirow{12}{*}{ 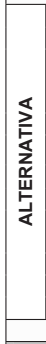 } & CASCARILLA DE HIERRO & 43.674 & 79.629 & 75.072 & 37.375 & 63.771 & 81.296 & 69.365 & 69.608 & 74.315 & 62.928 & 74.757 \\
\hline & CENIZAS & 1.582 .175 & 1.965 .184 & 2.313 .875 & 2.627 .593 & 3.004 .254 & 3.218 .882 & 3.452 .369 & 3.444 .422 & 3.247 .835 & 3.442 .580 & 2.245 .661 \\
\hline & $\begin{array}{l}\text { RESIDUOS DE } \\
\text { CONSTRUCCIÓN Y } \\
\text { DEMOLICIÓN } \\
\end{array}$ & & 2.838 & & & & & 962 & 24.625 & 6.761 & 908 & 23.012 \\
\hline & ESCORIAS & 267.161 & 964.125 & 522.494 & 448.651 & 351.220 & 489.971 & 831.273 & 1.725 .689 & 1.851 .258 & 1.768 .832 & 1.352 .021 \\
\hline & ESPUMA AZUCARERA & 44.399 & 46.696 & 27.250 & 7.490 & 78.605 & 114.179 & 98.468 & 52.971 & 40.320 & 33.030 & 42.360 \\
\hline & ESTÉRILES DE MINA & 110.711 & 146.637 & 126.895 & 135.425 & 87.717 & 135.187 & 78.516 & 78.001 & 81.702 & 67.715 & 20.307 \\
\hline & LODOS CARBONATO & 27.629 & 73.193 & 30.851 & 42.472 & 44.008 & 28.471 & 28.805 & 46.096 & 40.795 & 45.177 & 44.724 \\
\hline & $\begin{array}{l}\text { POLVO DE MÁRMOL Y OTRAS } \\
\text { INDUSTRIAS MINERALES } \\
\end{array}$ & 16.519 & 28.374 & 21.567 & 29.924 & 24.646 & 26.426 & 33.426 & 30.061 & 27.176 & 19.792 & 5.224 \\
\hline & SULFATO FERROSO & & & & & & 106.530 & 24.239 & 41.340 & 45.811 & 51.220 & 43.183 \\
\hline & YESO ARTIFICIAL & & & & & & 282.753 & 309.594 & 291.044 & 274.379 & 250.718 & 141.666 \\
\hline & OTROS & 21.117 & 13.953 & 27.262 & 28.256 & 25.374 & 24.738 & 21.315 & 22.122 & 26.812 & 72.193 & 152.925 \\
\hline & Total ALTE & 13.385 & 20.629 & 3.145 .266 & 3.357 .186 & 3.679 .595 & 08.433 & 4.948 .332 & 5.825.979 & 5.717 .164 & 5.815 .093 & 4.145 .840 \\
\hline & TOTAL & 46.623 .206 & 48.606 .392 & 49.835 .693 & 51.747 .739 & 53.792 .419 & 55.595 .035 & 58.824 .602 & 58.885 .755 & 59.204 .433 & 59.274 .608 & 50.609 .843 \\
\hline
\end{tabular}

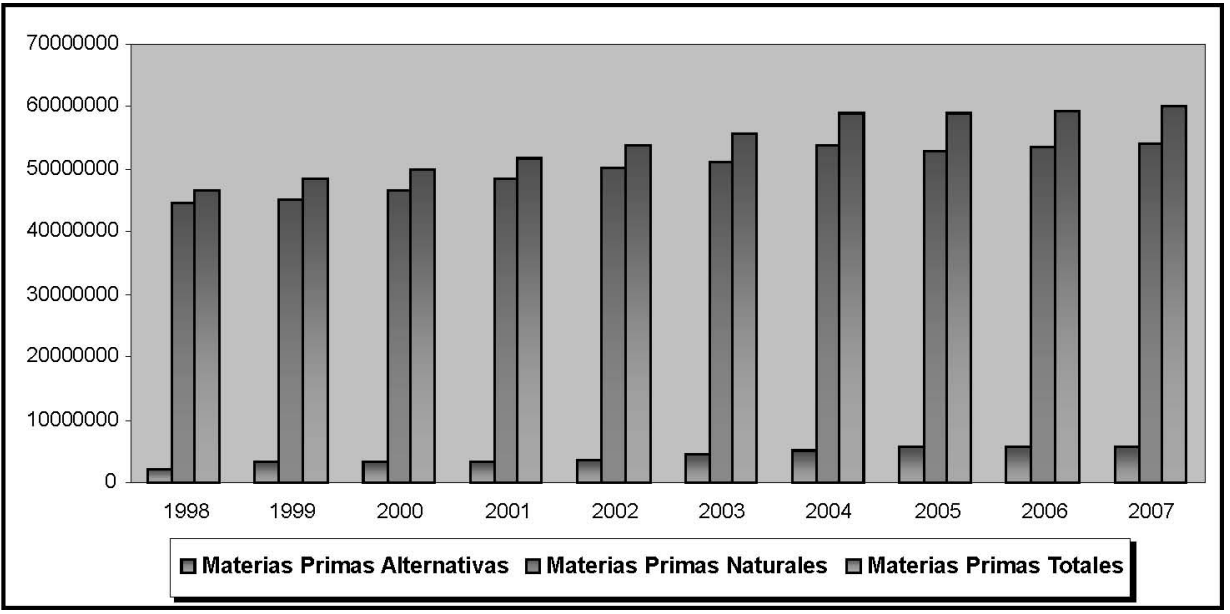

En su apuesta por la implantación de un circuito cerrado, la industria cementera también ha desarrollado la valorización energética, en la que se usan residuos para sustituir el combustible tradicional, aprovechando su energía calorífica.

Para obtener el clínker es necesario calentar las materias primas en grandes hornos rotatorios hasta su fusión parcial. El calor necesario para ello se obtiene de la combustión en una llama principal y, en ocasiones, en una secundaria. Este proceso requiere una gran cantidad de combustibles y aporta la posibilidad de valorizar ciertos residuos orgánicos y no orgánicos, utilizándolos como combustibles alternativos en sustitución de los tradicionales.
Esta práctica cobra cada vez mayor importancia en nuestro país, ya que representa una serie de ventajas, entre las que destacan (3):

-Evita el vertido de estos residuos y sus impactos asociados.

-Supone un tratamiento ecológico y seguro de los residuos, aprovechando al máximo su energía y minerales sin generar impactos añadidos sobre el entorno.

- Se ahorran combustibles fósiles no renovables (carbón y derivados del petróleo).

- Se disminuyen las emisiones globales, en particular las de $\mathrm{CO}_{2}$ (uno de los gases responsables del efecto invernadero) al sustituir combustibles fósiles por materiales que hubieran sido incinerados o fermentados
1. Consumo de materias primas (Fuente: Oficemen).

2. Evolución del consumo de materias primas (Fuente: Oficemen). 


\section{CONSUMO DE COMBUSTIBLES ALTERNATIVOS EN ESPAÑA}

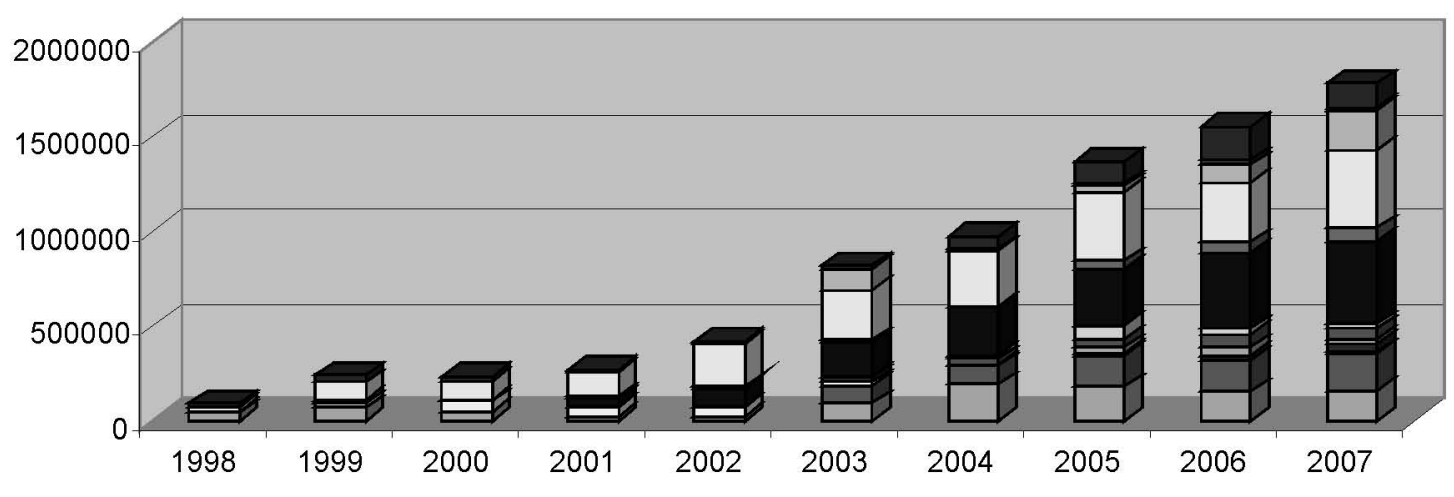

\begin{tabular}{|lll|}
\hline$\square$ Aceite usado & $\square$ Disolvente, barnices y pinturas & $\square$ Grasas animales \\
$\square$ Líquidos alternativos & $\square$ Otros & $\square$ Residuos industriales petróleo \\
$\square$ Celulosa o biomasa & $\square$ Lodos depuradora & $\square$ Harinas cárnicas \\
$\square$ Madera & $\square$ Neumáticos & $\square$ Otros combustibles \\
$\square$ Plásticos & $\square$ Serrín & \\
\hline
\end{tabular}

en vertederos, con sus correspondientes emisiones.

Aparte de estos beneficios, la valorización energética es una operación segura, limpia y eficiente que se inició hace varias décadas y se viene desarrollando con éxito en la mayoría de los países de la Unión Europea. Igualmente, se trata de un proceso sostenible al dar un tratamiento medioambientalmente correcto a los residuos y evitar la incineración o el vertido.

El sector cementero español utilizó en el año 2007 unas 350.000 toneladas de residuos como combustibles alternativos (Figura 3), que supusieron el $6,4 \%$ del consumo térmico de los hornos de clínker (2).

No obstante, el sector cementero español se encuentra a la cola de Europa en utilización de residuos como combustibles alternativos, mientras que países tradicionalmente "verdes" como Holanda y Suiza cuentan con unos niveles de sustitución muy elevados, $83 \%$ y $50 \%$ respectivamente. En Europa, aproximadamente el $70 \%$ de las fábricas de clínker emplean combustibles alternativos.

Sin embargo, para desarrollar más esta práctica medioambiental es preciso avanzar no sólo en cuanto a tecnología, sino también en otros campos. Así, en nuestro país su progreso depende en gran medida del apoyo de las Administraciones Públicas. En la actualidad, existen una serie de factores ajenos a la industria que dificultan el incremento de la utilización de combustibles alternativos, si bien la política de desarrollo sostenible del sector cementero contempla la colaboración con las Administraciones Públicas para el desarrollo de la valorización en España.

En definitiva, la valorización, tanto material como energética, es una de las claves en esta integración de los procesos productivos, lo cual permitirá reducir la dependencia de fuentes de energía extranjeras, disminuyento el consumo de combustibles fósiles, y minimizar las emisiones de Gases de Efecto Invernadero (GEI).

A modo de ejemplo, el uso de biomasa procedente de residuos ha producido mejoras medioambientales significativas que han supuesto un ahorro en tres años de 783.846 toneladas de $\mathrm{CO}_{2}$ mediante la sustitución de combustibles fósiles por este tipo de combustible alternativo (Figura 4).

En concreto, la tendencia de este ahorro fue de 220.403 toneladas de $\mathrm{CO}_{2}$ en el 2005, de 273.712 en el 2006 y de 289.731 en el 2007 (2).

\section{TRABAJO EN RED DE TODOS LOS SECTORES}

Todas las cuestiones anteriores están interrelacionadas con la filosofía de colaboración sectorial, ya que en la actualidad en todos los procesos industriales se busca la 
manera de utilizar los recursos en red. Es decir, buscar la configuración de la actividad industrial como un círculo en el que todo forme parte del proceso siguiente y sin que se deseche nada o lo mínimo posible.

Ejemplo de esta actitud en el sector cementero son los diferentes acuerdos que éste está firmando con otras industrias con el fin de reducir la generación de residuos. Así, la Agrupación de Fabricantes de Cemento de España (Oficemen) la Federación Española de la Recuperación (FER) y la Asociación Española para el Tratamiento Medioambiental de los Vehículos Fuera de Uso (Sigrauto) han acordado fomentar la valorización con los materiales obtenidos del tratamiento de los vehículos, electrodomésticos y otros productos cuando llegan al final de su vida útil.

El acuerdo prevé la colaboración para promover la valorización en las cementeras de parte de los residuos que se generan en las plantas fragmentadoras españolas donde los vehículos al final de su vida útil y otros muchos productos son sometidos a un proceso de triturado para poder separar y reciclar la parte metálica de los mismos. En España se dan de baja unos 700.000 turismos al año de cuyo peso total la legislación permite destinar hasta un $15 \%$ a vertederos convencionales, siendo ésta la peor solución medioambiental y, sin embargo, hasta hace poco la más practicada en España. No obstante, los nuevos acuerdos disminuirán sensiblemente esa cifra (7).

Al final de su vida útil, el usuario entrega el vehículo a un Centro Autorizado de Tratamiento para tramitar su baja y para que sean sometidos al proceso de descontaminación y retirada de aquellas piezas que sean susceptibles de ser reutilizadas. El resto del vehículo, normalmente compactado, es enviado a plantas fragmentadoras para continuar su proceso de tratamiento (Figura 5, pág. siguiente).

Las plantas fragmentadoras son grandes instalaciones en las que se trituran distintos tipos de materiales, principalmente vehículos fuera de uso descontaminados, grandes electrodomésticos y otros materiales férricos y no férricos para su posterior utilización como materias primas en procesos productivos.

En el proceso de fragmentación se utiliza una aspiración, una separación magnética y segregaciones manuales para apartar la parte férrica del resto de materiales.

Los residuos que se generan en las plantas de fragmentación de vehículos al final de su vida útil, una vez que han sido descontaminados y que serán objeto del presente proyecto, son:

a) Metales Férricos: se denomina comúnmente "chatarra fragmentada".

b) Residuo Ligero: el residuo ligero se obtiene del arrastre por corrientes de aire en el proceso de fragmentación en el molino de la fragmentadora (ver imagen). Este residuo está constituido por un conjunto heterogéneo de cauchos, gomas, polietilenos, plásticos acrílicos, etc. La composición es variable y diversa: $45 \%$ plásticos, $12 \%$ textiles, $32 \%$ de finos inertes y algo de madera, papel y metales.

Asimismo, pequeñas cantidades de metales, $\mathrm{Fe}, \mathrm{Cr}, \mathrm{Cu}, \mathrm{Mn}, \mathrm{Zn}$ y Ni, están presentes en concentraciones de partes por millón. El tamaño es variado, con un tamaño máximo que no excede en ningún caso de los 10 $\mathrm{cm}$, aunque esto dependerá del diseño del ciclón de la fragmentadora, y una parte más pequeña de alrededor de $1 \mathrm{~cm}$.

c) Residuo Pesado: este residuo está compuesto básicamente por una mezcla de gomas, plásticos, metales férricos y no férricos, fragmentos de madera y pequeños restos de hormigón proveniente de los contrapesos de los electrodomésticos de la línea blanca. Este residuo es enviado previo a cualquier otra operación a instalaciones de tratamiento por medios densos en los que se separan los metales férricos y no férricos del resto de materiales para su reciclaje.

Se han realizado pruebas para la utilización de la fracción ligera procedente de la fragmentación de vehículos fuera de uso (VFUs) como combustible alternativo en el quemador principal (Figura 6, pág. siguiente).

4. Toneladas de $\mathrm{CO}_{2}$ evitadas po a utilización de biomasa (Fuente: Oficemen).

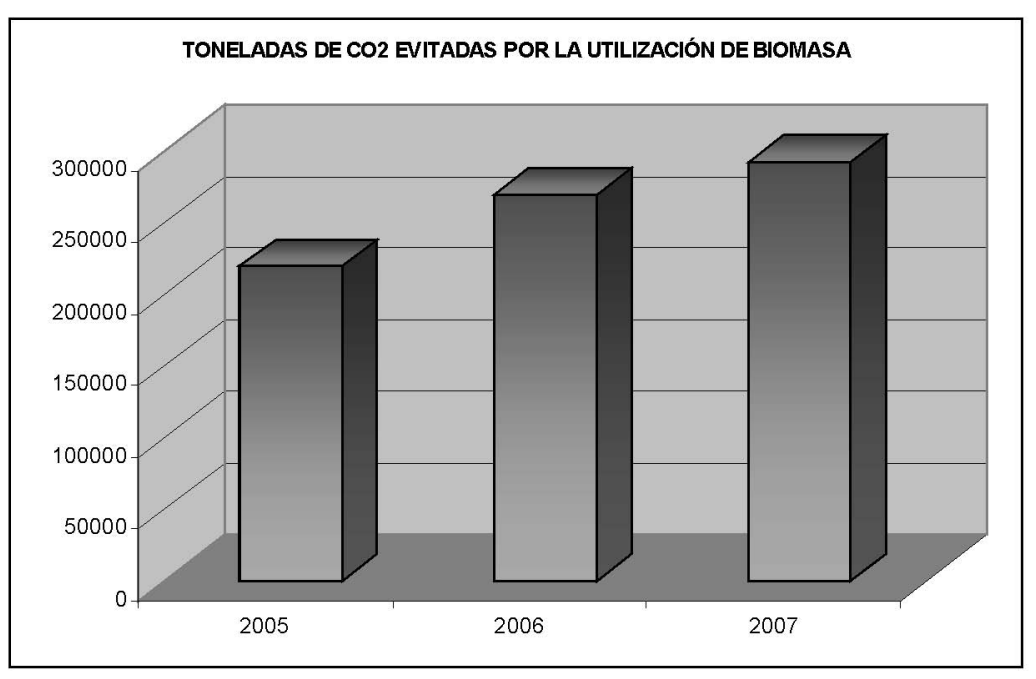




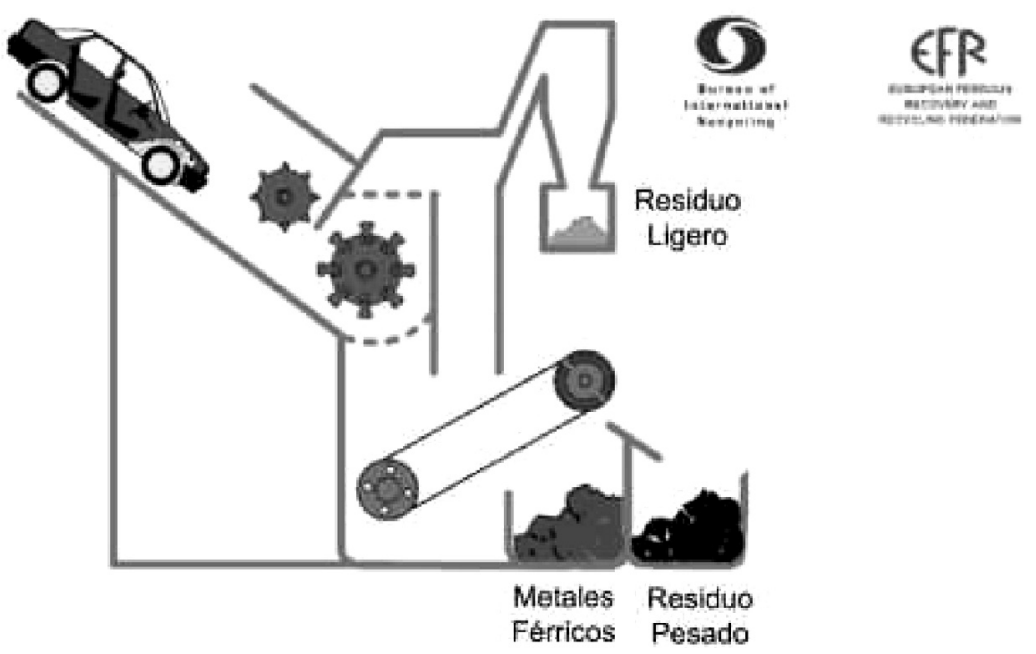

Metales Férricos

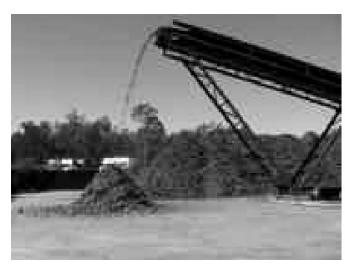

Residuo Pesado

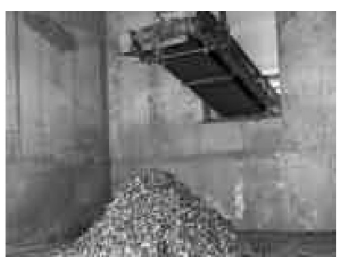

Residuo Ligero

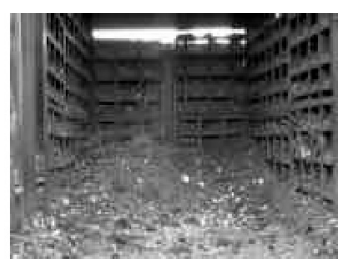

5. Proceso de fragmentación (Fuente: BIR).
Uno de los problemas principales fue gestionar con continuidad el residuo en la instalación debido a la heterogeneidad y gran contenido en alambres, trozos metálicos, tierra y vidrio, que producen continuos atascos en la inyección y gran desgaste en las cuchillas, rejilla del triturador y tubería de inyección.

El máximo caudal alimentado al horno ha variado entre los 500 kg/h y 1.000 kg/h en función de la planta donde se estuviese produciendo la valorización. Las fábricas disponen de mediciones de dioxinas y furanos alimentando este tipo de residuo al quemador, no habiéndose obtenido un aumento en la emisión de este contaminante con respecto a la utilización de otro tipo de combustibles.

El resto de las emisiones monitorizadas en continuo, $\mathrm{SO}_{2}, \mathrm{NOx}, \mathrm{HCl}, \mathrm{HF}, \mathrm{CO}, \mathrm{CO}_{2}$, COT, no se ven afectadas por la utilización de este tipo de combustible manteniéndose en los niveles normales de emisión para el horno.

Por otro lado, Oficemen y la Unión de Empresas Siderúrgicas (Unesid) también han unido sus esfuerzos para impulsar el uso de residuos en la fabricación del cemento a través de un acuerdo de colaboración. Éste supone la sustitución de parte de las materias primas utilizadas habitualmente en la producción de clínker por escorias de acería de horno. Así, se podría reducir el impacto producido por la extracción de las canteras, ya que estos residuos aportan el hierro necesario para fabricar el clínker.

En España funcionan actualmente 25 acerías eléctricas, cuya producción en 2006 se situó en 14,8 millones de toneladas de acero. Las escorias generadas en el proceso siderúrgico, más de dos millones de toneladas/año, están catalogadas como residuos no peligrosos. El correcto tratamiento de los mismos supone una serie de beneficios de carácter global, tanto desde el punto de vista de los generadores como desde el social, puesto que supone una disminución de los costes de vertido, un ahorro de materias primas, la reducción del impacto medioambiental y el incremento de la calidad de vida en el entorno de estas industrias (8).

Por este motivo, este tipo de colaboración es necesaria, al igual que la apuesta por la innovación, que ha ido de la mano del desarrollo de sistemas respetuosos con el medio ambiente, con el fin de compatibilizar la evolución industrial con el cuidado del entorno.

A nivel europeo se ha puesto en marcha un proyecto a través del que se realizará una reflexión en busca del "Residuo 0". Se trata del proyecto Pressure, una iniciativa que desde el punto de vista intelectual tratará de reunir en torno a la idea del reciclado de residuos a todos los sectores europeos a través de un trabajo de investigación de las diferentes iniciativas, políticas, tecnologías, acuerdos, etc. existentes en todos los países miembro.

\section{CONCLUSIÓN}

Al hablar de sostenibilidad medioambiental automáticamente se centra el tema en la emisión de GEl y, en concreto, de $\mathrm{CO}_{2}$, ya que es el cambio climático la principal preocupación del siglo XXI. Sin embargo, es preciso centrarse en las causas de dichas emisiones, que simplemente son el resultado del proceso industrial, siendo necesario disminuir la extracción de materias primas naturales y la quema de combustibles fósiles. La reincorporación de los residuos al proceso productivo para su reciclado, valorización material y energética es una de las principales vías técnicamente viables para la reducción de emisiones.

Igualmente, y teniendo en cuenta la aportación del sector cementero a esta causa, es preciso destacar que en los últimos años se ha desarrollado una importante labor de investigación e implantación de sistemas que 
potencien la sostenibilidad de los procesos productivos. Cada día hay más avances que permiten ir hacia una industria más limpia y con menor afección en el entorno. No obstante, es preciso ir más allá y apostar por un trabajo cooperativo de todos los sectores industriales, consiguiendo una producción en circuito cerrado en la que se eviten los residuos, se ahorre energía y se reduzcan las emisiones de GEI.

En este sentido, el cometido actual de la industria es desarrollar una configuración de su actividad en círculo, consiguiendo la reutilización de materiales y energía procedentes de unos procesos en otros, independientemente del sector al que se dediquen.

Pero aún hay más, es igualmente necesario desarrollar teóricamente este concepto de producción en cadena, ya que únicamente de esta manera conseguiremos realmente extender esta filosofía, estableciendo unos criterios básicos para su implantación, al tiempo que se podrían definir las Mejores Técnicas Disponibles para ello.

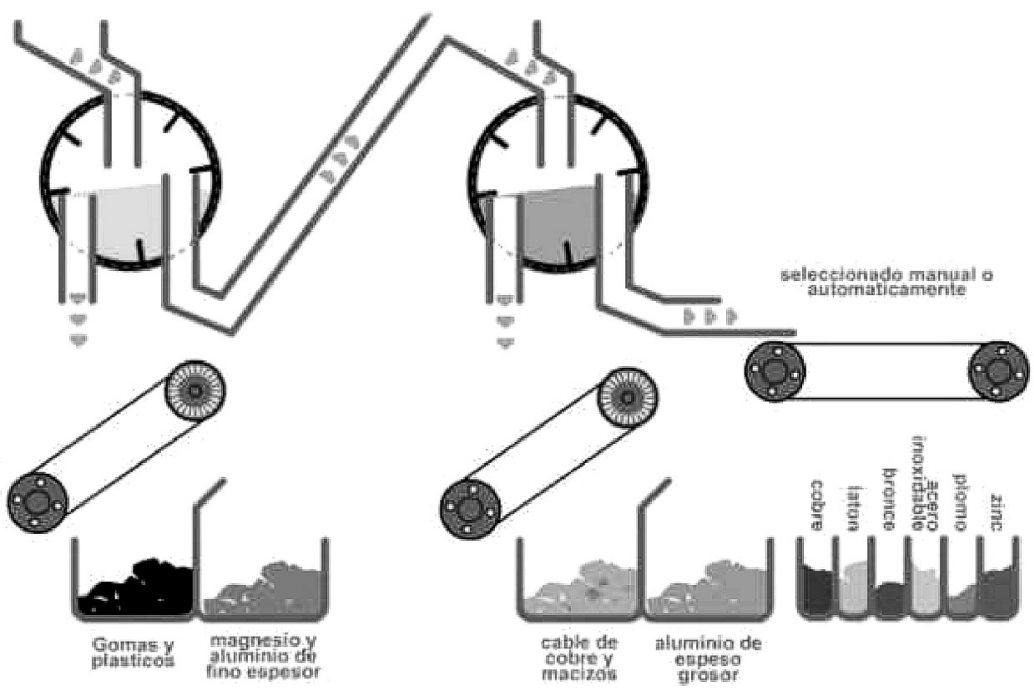

Porque ya no es suficiente con ser una industria eficiente, saneada y sostenible, sino que debemos ser una industria unida, cooperativa y en circuito cerrado para contribuir de la mejor manera posible a la sostenibilidad.

\section{REFERENCIAS}

(1) "El ciclo de vida del cemento. Un puente a la sostenibilidad en la construcción". Cemento Hormigón, no 915 (2008), pp. 66-75.

(2) Oficemen. "Anuario 2007". Madrid, 2008

(3) Oficemen. "Anuario 2006". Madrid, 2007.

(4) Fundación del Cemento y el Medio Ambiente (Cema). "Informe Valorización de Residuos en la Industria Cementera Europea (VRICE)". Madrid, 2007.

(5) Ministerio de Industria. "Plan Nacional de Asignación de derechos de emisión 2005-2007". Madrid, 2005.

(6) World Business Council for Sustainable Development. "The Cement Sustainability Initiative". Madrid, 2007.

(7) Oficemen, FER y Sigrauto. "Acuerdo Marco FER-Oficemen y Sigrauto para la valorización energética de residuos". Madrid, 2007.

(8) Oficemen y Unesid. "Acuerdo Marco Unesid y Oficemen para el aprovechamiento de materiales procedentes del sector siderúrgico". Madrid, 2007.

(9) www.autocemento.com.

(10) www.bir.org.

(11) www.efr2.org. 


\section{Publicaciones del Instituto Eduardo Torroja-CSIC}

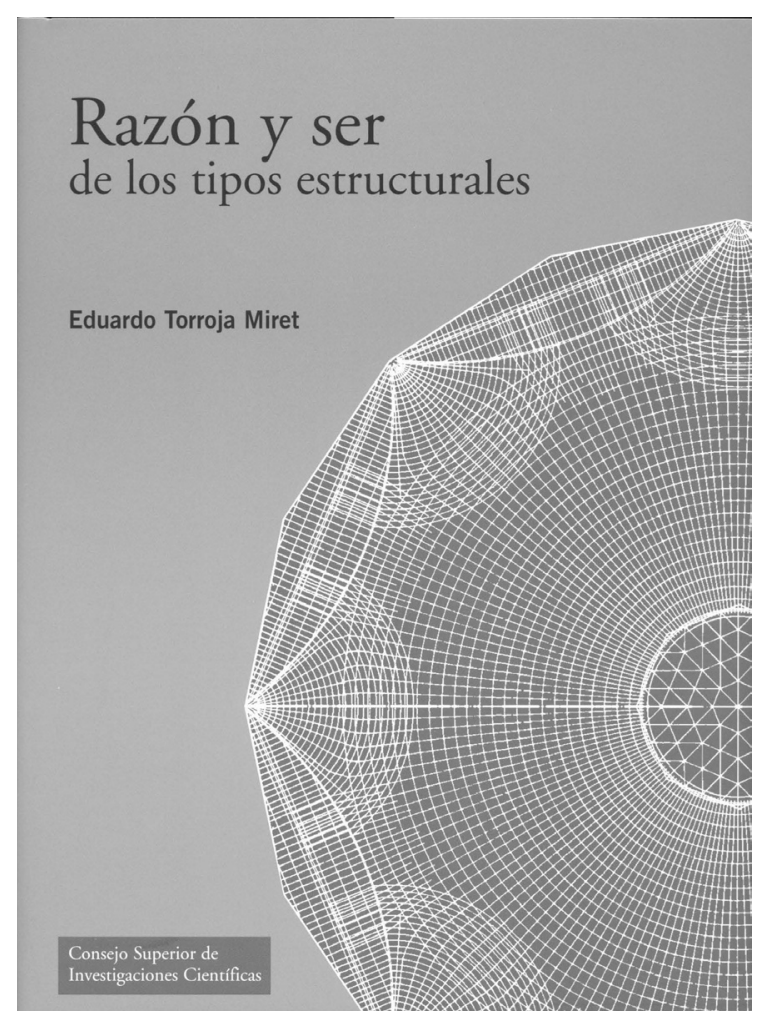

Cada material tiene una personalidad específica distinta, y cada forma impone un diferente fenómeno tensional. La solución natural de un problema -arte sin artificio-, óptima frente al conjunto de impuestos previos que la originaron, impresiona con su mensaje, satisfaciendo, al mismo tiempo, las exigencias del técnico y del artista. El nacimiento de un conjunto estructural, resultado de un proceso creador, fusión de técnica con arte, de ingenio con estudio, de imaginación con sensibilidad, escapa del puro dominio de la lógica para entrar en las secretas fronteras de la inspiración. Antes y por encima de todo cálculo está la idea, moldeadora del material en forma resistente, para cumplir su misión.

A esa idea va dedicado este libro.

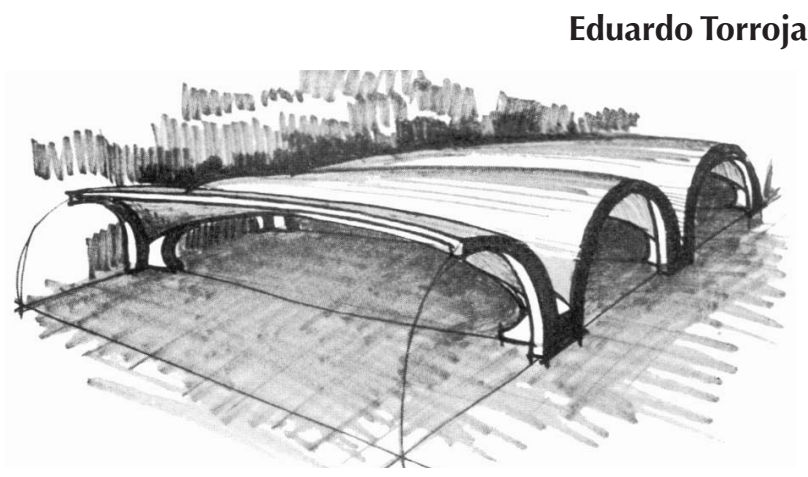

Razón y ser de los tipos estructurales alcanza en la Colección «Textos Universitarios» su tercera edición, revisada en 2007 . Las anteriores fueron publicadas sucesivamente por el Consejo Superior de Investigaciones Científicas a través del Instituto de Ciencias de la Construcción «Eduardo Torroja», que lleva el nombre de su autor.

El prestigio internacional de Eduardo Torroja Miret dentro de la Ingeniería como creador e innovador en el campo de las estructuras, es bien conocido. Esta capacidad excepcional de creación, interpretación e innovacíón queda perfectamente reflejada en este libro.

Obra fundamental de notable contenido formativo y didáctico para los estudiantes y estudiosos de la Arquitectura y la Ingeniería, Razón y Ser mantiene, a pesar del tiempo transcurrido desde su edición original, plena vigencia.

Este hecho y la demanda continua de ejemplares, dentro y fuera de España, nos ha animado a su reedición en una colección que como «Textos Universitarios» se plantea llevar a sus lectores obras fundamentales de permanente demanda, y nuevas aportaciones igualmente importantes tanto por la novedad de sus contenidos, como por la solidez y actualidad de los mismos.

Finalmente añadir que esta nueva edición de Razón y Ser mejora, a nuestro juicio, la calidad,editorial y presentación de las anteriores, y esperamos obtenga la misma acogida de las que la precedieron.

\section{VENTA}

Departamento de Publicaciones

Vitruvio, 8. 28006 Madrid

Sección de Revistas. Tfnos: 9156128 33/91 51597 42-44-17; Fax: 915629634

e-mail: publ@orgc.csic.es 